\title{
Simple frequency-dependent tools for analysis of inherent control limitations
}

\author{
SIGURD SKOGESTAD †‡, MORTEN HOVD $\dagger$ and \\ PETTER LUNDSTRÖM†
}

Keywords: Dynamic resilience, relative gain array, disturbance rejection, process control

By a plant's inherent control limitations we mean the characteristics of the plant which will cause poor control performance irrespective of what controller is used. Another frequently used term is 'dynamic resilience' which is the best closed-loop performance achievable using any controller. Since a plant's dynamic resilience cannot be altered by change of the control algorithm, but only by design modifications, it follows that the term controllability provides a link between process design and process control. In this paper we focus on two aspects of controllability. The plants' sensitivity to disturbances and the limitations imposed by interactions when using decentralized control. We use simple tools such as the RGA, the PRGA (Performance RGA) and the closely related Closed Loop Disturbance Gain (CLDG). For example, if $k$ th column of the CLDG is large, then this indicates that disturbance $k$ will be difficult to reject. This may pinpoint the need for modifying the process. The PRGA provides a measure of interaction which also includes one-way coupling. In the paper we apply these measures to distillation column control and fluid catalytic cracker (FCC) control.

\section{Introduction}

In this paper we study simple tools for evaluating a plant's inherent control characteristics. We will refer to this as the 'dynamic resilience' (Morari, 1983) of the plant and use the following definition:

Dynamic resiliency (of a plant) is the (best) quality of the response which can be obtained for the plant by use of feedback control.

Admittedly, this definition is not very precise, since, for example, 'best' is not defined. Closely related terms are 'dynamic operability' and 'achievable performance'. Also the term 'controllability' is often used in the meaning of 'dynamic resilience' (e.g., Perkins, 1989). This use of the term is in accordance with engineering practice, where a plant is called 'controllable' if it is possible to achieve the specified aims of the control, whatever these may be (Rosenbrock, 1970, p. 161). Unfortunately, the term 'controllability' also has a well established definition in terms of Kalman's state controllability. The term state controllability (and state observability) is mainly related to realization theory and has little to do with the engineering term controllability. If we have a minimal realization of a system (fewest number of states) then all states are controllable and observable. Noncontrollable and nonobservable states correspond to those which

Received 20 December 1991.

$\dagger$ Chemical Engineering, University of Trondheim, NTH, N-7034 Trondheim, Norway.

$\ddagger$ Author to whom correspondence should be addressed. E-mail: skoge@,kjemi.unit.no, Phone: 47-7-594154, Fax: 47-7-591410. 
disappear by exact pole-zero cancellation when we evaluate the transfer function. We will therefore not use the term 'controllability' in this paper, although it would have been the preferred term to use if it did not already have this rather restrictive definition.

A key idea in the term dynamic resilience is that it is an inherent property of the plant, and is independent of the selected controller parameters (it is assumed that the optimal tunings are used). Of course, one may restrict the class of allowed controllers, and consider, for example, 'dynamic resilience using linear controllers' (which we do throughout this paper) or 'dynamic resilience using decentralized control' (which we consider in most parts of this paper). Since the dynamic resilience cannot be altered by change of the control algorithm, but only by design modifications, it follows that the term dynamic resilience provides a link between process design and process control. 'Design modifications' of course include modifications of the process units (eg., adding buffer tanks, increasing the number of trays in a column), but they also include selection of control objectives (e.g., control temperature or composition), selection of manipulated variables (e.g., placing of bypass streams) and selection of measurements. Stephanopolous (1984, p. 512) gives some examples of how process modifications may change the dynamic resilience. Seborg et al. $(1989$, p. 682) give some guidelines for the control structure selection, that is, selection of controlled, manipulated and measured variables.

What limits dynamic resilience? Perfect control can only be achieved if the plant is invertible (Morari, 1983). Several authors (e.g., Rosenbrock, 1970) discuss the deteriorating effect of Right-half plane (RHP) zeros and time delays which make it impossible to invert the plant and retain stability of the closed-loop system. Constraints make it impossible to implement in practice the inputs that perfect inversion requires. Model uncertainty results in mismatch between the model used by the controller and the actual plant. Large RGA-elements imply sensitivity to model uncertainty for multivariable systems (Skogestad and Morari, 1987 b). All these items generally imply that the speed of the response (bandwidth) cannot be too high.

In this paper we will focus on two additional aspects of dynamic resilience: The plants sensitivity to disturbances and the limitations imposed by interactions when using decentralized control. The requirement of disturbance rejection requires the speed of response (bandwidth) to be high, and is thus generally in conflict with the requirements imposed by RHP-zeros, time delays, model uncertainty and constraints.

We will concentrate on simple measures which depend on the plant model only such as the RGA, PRGA and CLDG. The advent of the computer has largely removed the need to develop simplified tools in order to save computation time. However, there is still a need for simple tools to yield insight and to assist the engineer in prescreening the large number of alternative control structures, and to get initial estimates of a system's dynamic resilience. For example, the RGA is an ideal tool in this respect; it may be computed using only limited information and one calculation is sufficient for screening a large number of alternatives.

However, there are of course limitations with such simple tools, and more powerful and exact methods must be used after the initial screening. Such methods generally involve performing a controller design. For example, one method is to obtain an upper bound on performance by adjusting the performance weight such that the optimal controller satisfies the performance objective. One specific approach, which also takes model uncertainty into account, is to adjust the performance weight such that the structured singular value $(\mathrm{mu})$ for robust performance is 1 (e.g., see 'Approach 2' in Skogestad and Lundström, 1990). 


\section{Some simple tools: RGA, PRGA and CLDG}

Notation. Let $y(s)$ denote the output response and let $e(s)=y(s)-r(s)$ denote the output error. The closed loop response to a setpoint $r$ and a disturbance $z$ becomes

$$
e(s)=-S(s) r(s)+S(s) G_{d}(s) z(s) ; \quad S=(I+G C)^{-1}
$$

The Laplace variable $s$ is often omitted to simplify notation. $G$ is assumed to be a $n \times n$ square matrix, but $G_{d}$ may be nonsquare. In most of this paper we consider decentralized control, and the controller $C(s)$ is diagonal with entries $c_{i}(s)$ (see Fig. 1). This implies that after the variable pairing has been determined, the order of the elements in $y$ and $u$ has been arranged so that the plant transfer matrix $G(s)$ has the elements corresponding to the paired variables on the main diagonal.

$R G A$. The RGA was first introduced by Bristol (1966) as a measure of interaction and as a tool for pairing selection for decentralized control. However, later it has become clear that the RGA is a useful controllability measure also when decentralized control is not used (e.g., Skogestad and Hovd, 1990). The RGA was originally defined at steady-state, but it may easily be extended to higher frequencies (Bristol, 1978). Shinskey $(1967,1984)$ has demonstrated practical applications of the steady-state RGA. The book by McAvoy (1983) yields a good introduction to interactions in multivariable systems, and demonstrates the usefulness of the frequency-dependent RGA.

For a $2 \times 2$ plant $G(s)$ the RGA-matrix is

$$
\begin{aligned}
& \Lambda(s)=\left(\begin{array}{ll}
\lambda_{11} & \lambda_{12} \\
\lambda_{21} & \lambda_{22}
\end{array}\right)=\left(\begin{array}{cc}
\lambda_{11} & 1-\lambda_{11} \\
1-\lambda_{11} & \lambda_{11}
\end{array}\right) \\
& \lambda_{11}=\frac{1}{1-Y} ; \quad Y(s)=\frac{g_{12} g_{21}}{g_{11} g_{22}}(s)
\end{aligned}
$$

where $Y$ is the interaction measure introduced by Balchen (1958). For $n \times n$ plants the RGA is defined by the ratio of the 'open-loop' and 'closed-loop' gains between input $j$ and output $i$

$$
\lambda_{i j}(s)=\frac{\left(\partial y_{i} / \partial u_{j}\right)_{u_{l \neq j}}}{\left(\partial y_{i} / \partial u_{j}\right)_{y_{l \neq i}}}=g_{i j}(s)\left[G^{-1}(s)\right]_{j i}
$$

Thus, a RGA matrix can be computed using the formula

$$
\Lambda(s)=G(s) \times\left(G^{-1}(s)\right)^{T}
$$

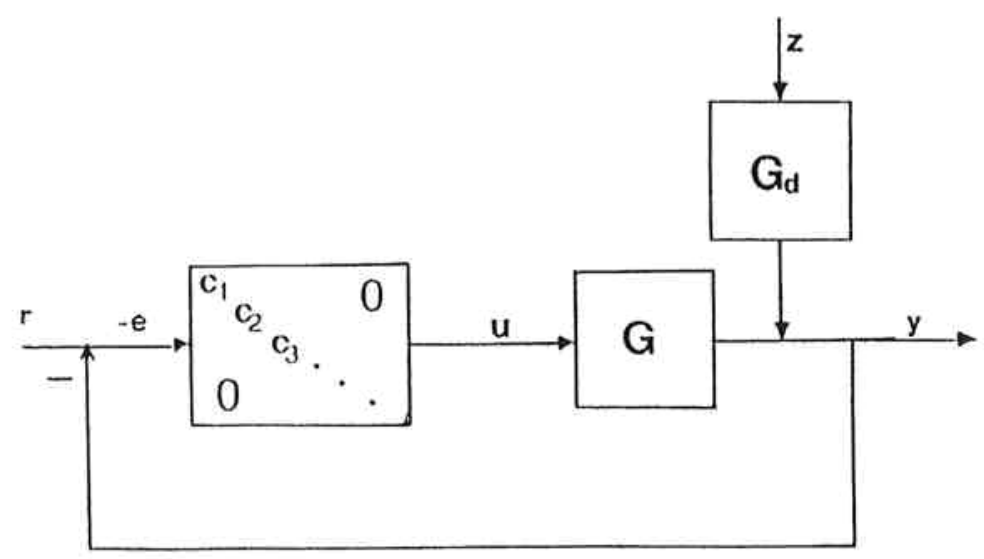

Figure 1. Block diagram of decentralized control structure. 
where the $\times$ symbol denotes element by element multiplication (Hadamard or Schur product). The RGA matrix has some interesting algebraic properties (Bristol, 1966):

(a) It is scaling independent (e.g., independent of units chosen for $u$ and $y$ ). Mathematically, $\Lambda\left(D_{1} G D_{2}\right)=\Lambda(G)$ where $D_{1}$ and $D_{2}$ are diagonal matrices.

(b) All row and column sums equal one.

(c) Any permutation of rows or columns in $G$ results in the same permutations in the RGA.

(d) If $G(s)$ is triangular then $\Lambda(G)=I$.

Another important usage of the RGA is that pairing on negative steady-state relative gains should be avoided. The reason is that with integral control this yields instability of either (1) the overall system, (2) the individual loop, or (3) the remaining system when the loop in question is removed. It is also established that plants with large RGA-values, in particular at high frequencies, are fundamentally difficult to control irrespective of the controller used (poor dynamic resilience).

$P R G A$. One inadequacy of the RGA (e.g., McAvoy, 1983, p. 166) is that it, because of property $(d)$, may indicate that interactions are no problem, but significant one-way coupling may exist. To overcome this problem we introduce the performance relative gain array (PRGA). The PRGA-matrix is defined as

$$
\Gamma(s)=G_{\text {diag }}(s) G(s)^{-1}
$$

where $G_{\text {diag }}(s)$ is the matrix consisting of only the diagonal elements of $G(s)$, i.e., $G_{\text {diag }}=\operatorname{diag}\left\{g_{i i}\right\}$. This matrix was originally introduced at steady-state by Grosdidier (1990) in order to understand the effect of directions under decentralized control, and similar ideas are presented by Friedly (1984). We will derive its relationship to performance in Section 6. The elements of $\Gamma$ are given by

$$
\gamma_{i j}(s)=g_{i i}(s)\left[G^{-1}(s)\right]_{i j}=\frac{g_{i i}(s)}{g_{j i}(s)} \lambda_{j i}(s)
$$

Note that the diagonal elements of RGA and PRGA are identical, but otherwise PRGA does not have all the nice algebraic properties of the RGA. PRGA is independent of input scaling, that is, $\Gamma\left(G D_{2}\right)=\Gamma(G)$, but it depends on output scaling. This is reasonable since performance is defined in terms of the magnitude of the outputs. Note that PRGA $=G_{s}^{-1}$ where $G_{s}$ is obtained by input scaling of $G$ such that all the diagonal elements are 1 (at all frequencies).

CLDG. A closely related measure, the closed loop disturbance gain (CLDG), was recently introduced by Skogestad and Hovd (1990). For a disturbance $k$ and an output $i$, the CLDG is defined by

$$
\delta_{i k}(s)=g_{i i}(s)\left[G(s)^{-1} G_{d}(s)\right]_{i k}
$$

The reason for the name CLDG will become clear later. A matrix of CLDG's may be computed from

$$
\Delta=\left\{\delta_{i k}\right\}=G_{\mathrm{diag}} G^{-1} G_{d}=\Gamma G_{d}
$$

The CLDG is scaling dependent, as it depends on the expected magnitude of disturbances and outputs. The CLDG is closely related to the relative disturbance gain (RDG), denoted $\beta_{i k}$, introduced by Stanley et al. (1985). We have $\delta_{i k}(s)=\beta_{i k}(s) g_{d i k}(s)$. 
Note that the PRGA and CLDG have to be recomputed whenever another choice of pairings is selected, whereas the RGA need only be rearranged in accordance with the rearrangement of $G$ (because of property c).

\section{Scaling}

The RGA has the advantage of being scaling dependent, but for the other measures it is crucial that the variables are scaled properly. In general, the variables should be scaled to be within the interval -1 to 1 , that is, their desired or expected magnitudes should be normalized to be less than 1 . Recommended scalings:

- Inputs (u): An $u_{j}$ of magnitude 1 should correspond to the largest allowed input signal e.g., the input reaching its constraint (i.e., the inputs are normalized with respect to their allowed range).

- Outputs (y): An $e_{i}$ of magnitude 1 should correspond to the largest allowed control error (i.e., the outputs are normalized with respect to their allowed range).

- Disturbances (z): $\mathrm{A} z_{k}$ of magnitude 1 should correspond to the largest expected disturbance (i.e., the disturbances are normalized with respect to their expected range).

The measures depend on scaling as follows: RGA: independent of scaling; PRGA: depends on scaling of $y ; G_{d}$, CLDG and RDG: depends on scaling of $z$ and $y$; Condition number and Disturbance condition number: depends on scaling of $u$ and $y$. All interpretations and examples in this paper assume that appropriate scaling has been performed.

Comment. We do not here separately scale the setpoints $r_{i}$, and therefore implicitly assume that these are of the same magnitude as the allowed errors. If this is not the case, then one should use the diagonal matrix $D_{r}$ to scale the setpoints such that they all are of magnitude 1, and use the matrix $P R G A_{r}=G_{\text {diag }} G^{-1} D_{r}$ to evaluate the performance for setpoint tracking.

\section{Performance requirements imposed by disturbances}

Some plants have better 'built-in' disturbance rejection capabilities than others, that is, their dynamic resilience with respect to disturbance rejection is better. For single-input single-output (SISO) systems, the sensitivity to disturbances is directly given by $g_{d}(s)$ (we use a lower-case letter to denote scalar transfer function). We have when we consider only disturbance rejection $(r=0)$

$$
\boldsymbol{e}(s)=y(s)=S(s) g_{d}(s) z(s)
$$

If we assume that scaling has been applied to $g_{d}$ such that at each frequency the expected $z(j \omega)$ is less than 1 in magnitude, then the requirement to achieve $e(j \omega)$ less than 1 in magnitude is that at each frequency

$$
\left|S(j \omega) g_{d}(j \omega)\right|<1 \quad \text { or }|S(j \omega)|<1 /\left|g_{d}(j \omega)\right|
$$

This is a performance requirement on the sensitivity function $S$ imposed by disturbance rejection. If $\left|g_{d}\right|$ is large (larger than 1) then feedback is needed to reject this disturbance. A plant with a small $g_{d}$ is preferable (better dynamic resilience) since the need for feedback control then is less, or alternatively, with a given feedback controller (given $S$ ) 
the effect on $e$ of the disturbance is less. At low frequencies, within the bandwidth $\omega_{B}$, we have $S \approx(g c)^{-1}$ and the disturbance requirement (10) yields the following constraint on the loop transfer function $g c$

$$
|g c|>\left|g_{d}\right| \forall \omega<\omega_{B}
$$

Example (see Fig. 2): Assume that the appropriately scaled $g_{d}(s)=k_{d} /\left(1+\tau_{d} s\right)$, and assume $k_{d}>1$. Then the required bandwidth, $\omega_{B}$, imposed by the requirement of disturbance rejection, is the frequency at which the asymptote of $\left|g_{d}(j \omega)\right|$ is 1 . We get $\omega_{B}=k_{d} / \tau_{d}$. We want the required $\omega_{B}$ to be small. That is, we get the obvious result that a 'large' ( $k_{d}$ large) and 'fast' ( $\tau_{d}$ small) disturbance requires a large bandwidth and is difficult to reject.

For multivariable systems we get

$$
e=S G_{d} z \approx(G C)^{-1} G_{d} z
$$

where the approximation holds at low frequencies where control is effective and $S$ is small. One may generalize the use of $\left|g_{d}\right|$ for SISO systems to multivariable systems by simply using the singular values of $G_{d}$. The frequency range where the singular values of $G_{d}$ cross 1, will give the required bandwidth region for the closed-loop system. However, for multivariable systems one should also consider the directions of $G_{d}$ relative to those of $G$. These issues are discussed by Skogestad and Morari (1987 a) who introduce the disturbance condition number. The special case for multivariable system when the controller is diagonal is discussed in detail in the next section on decentralized control.

\section{Performance relationships for decentralized control}

For SISO systems we found at low frequencies that the loop transfer function $g c$ must be larger than $g_{d}$ in magnitude to achieve disturbance rejection. The objective of this chapter is to derive similar derive bounds on each loop transfer functions, $g_{i i} c_{i}$, for the case with decentralized control. Essentially, we want to find the apparent disturbance gain for an individual loop $i$ when using decentralized control. $\dagger$ We will show that the answer to this problem is given by the closed loop disturbance gains (CLDG), which are equal to the PRGA-matrix multiplied by the open-loop disturbance gains $G_{d}$. We will

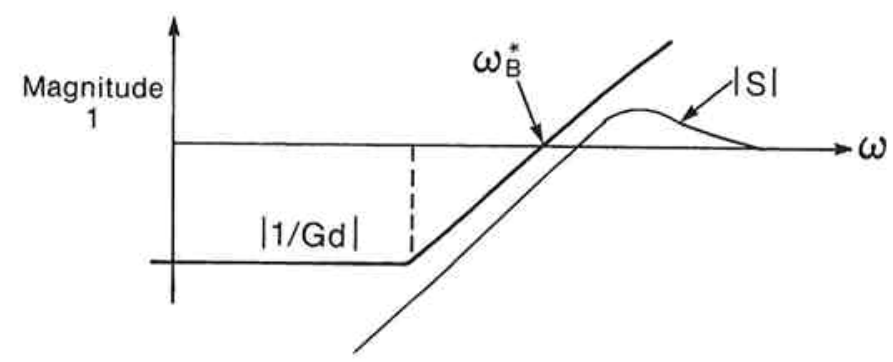

Figure 2. Performance restrictions imposed by the requirement of disturbance rejection for single-input single-output system. To reject a disturbance with model $G_{d}(s)=k_{d} /\left(1+\tau_{d} s\right)$ the closed-loop bandwidth must be higher than $\omega_{B}^{*}=k_{d} / \tau_{d}$.

†It may seem that this may be obtained by simply closing all the other loops with perfect control and consider the effective disturbance gain to the uncontrolled output $y_{i}$. However, this is not the case as this assumes that the input $u_{i}$ is constant, while we want to use $u_{i}$ to keep the output $y_{i}$ constant. 
also consider setpoints and find that the PRGA-matrix enters in a similar way also here.

Scaling is essential when evaluating performance and we stress that all interpretations given in this section assume that $G$ and $G_{d}$ have been scaled as discussed in Section 3.

Consider the effect of a setpoint change $r_{j}$ and a disturbance $z_{k}$ on the offset $e_{i}$. With all loops closed the closed-loop response becomes (Fig. 1)

$$
\boldsymbol{e}_{i}=-[S]_{i j} r_{j}+\left[S G_{d}\right]_{i k} z_{k}
$$

or on matrix form

$$
\boldsymbol{e}=-S R+S G_{d} z
$$

For $\omega<\omega_{B}$ we may usually assume $S=(I+G C)^{-1} \approx(G C)^{-1}$. Provided the corresponding cofactor of $G$ is nonzerot, and $c_{i}$ is sufficiently large (decentralized control), this approximation will also hold for individual elements

$$
[S]_{i j} \approx \frac{\left[G^{-1}\right]_{i j}}{c_{i}} ; \quad\left[S G_{d}\right]_{i k} \approx \frac{\left[G^{-1} G_{d}\right]_{i k}}{c_{i}} ; \quad \omega<\omega_{B}
$$

With this approximation (13) becomes

$$
e_{i} \approx-\left[G^{-1}\right]_{i j} \frac{1}{c_{i}} r_{j}+\left[G^{-1} G_{d}\right]_{i k} \frac{1}{c_{i}} z_{k} ; \omega<\omega_{B}
$$

If $g_{i i}(s) \neq 0$ the definitions of the PRGA and CLDG yield

$$
\boldsymbol{e}_{i} \approx-\frac{\gamma_{i j}}{g_{i i} c_{i}} r_{j}+\frac{\delta_{i k}}{g_{i i} c_{i}} z_{k} ; \quad \omega<\omega_{B}
$$

Using $S_{\mathrm{diag}}=\left(I+G_{\mathrm{diag}} C\right)^{-1} \approx \operatorname{diag}\left\{1 /\left(g_{i i} c_{i}\right)\right\}$ this may be written on matrix form

$$
e \approx-S_{\mathrm{diag}} G_{\mathrm{diag}} G^{-1} r+S_{\mathrm{diag}} G_{\mathrm{diag}} G^{-1} G_{d} z=-S_{\mathrm{diag}} \Gamma r+S_{\mathrm{diag}} \Delta z ; \quad \omega<\omega_{B}
$$

From (17) we see that the ratio $\gamma_{i j} /\left(g_{i i} c_{i}\right)$ gives the magnitude of the offset in output $i$ to a setpoint change in output $j$. This ratio should preferably be small. That is, on a conventional magnitude Bode plot, the curve for $\left|\gamma_{i j}\right|$ should lie below $\left|g_{i i} c_{i}\right|$ at frequencies where we want small offsets.

For process control disturbance rejection is usually more important than setpoint tracking. From (17) we see the ratio $\delta_{i k} /\left(g_{i i} c_{i}\right)$ gives the magnitude of the offset in output $i$ to a disturbance $z_{k}$. That is, the curve for $\left|\delta_{i k}\right|$ should lie below $\left|g_{i i} c_{i}\right|$ at frequencies where we want small offsets. A plot of $\left|\delta_{i k}(j \omega)\right|$ will give useful information about which disturbances $k$ are difficult to reject.

Note that for input disturbances $G_{d}=G$ and we get $\delta_{i k}=g_{i i}$. Thus, large diagonal elements in $G$ (when appropriately scaled) may imply difficulties rejecting input disturbances.

$\dagger$ Cofactors of $G$ identically equal to zero are relatively rare except for the offdiagonal zero elements of triangular transfer matrices. However, for these zero elements both $[S]_{i j}$ and $\left[(G C)^{-1}\right]_{i j}$ are zero and the approximation holds even though the cofactor is zero. 

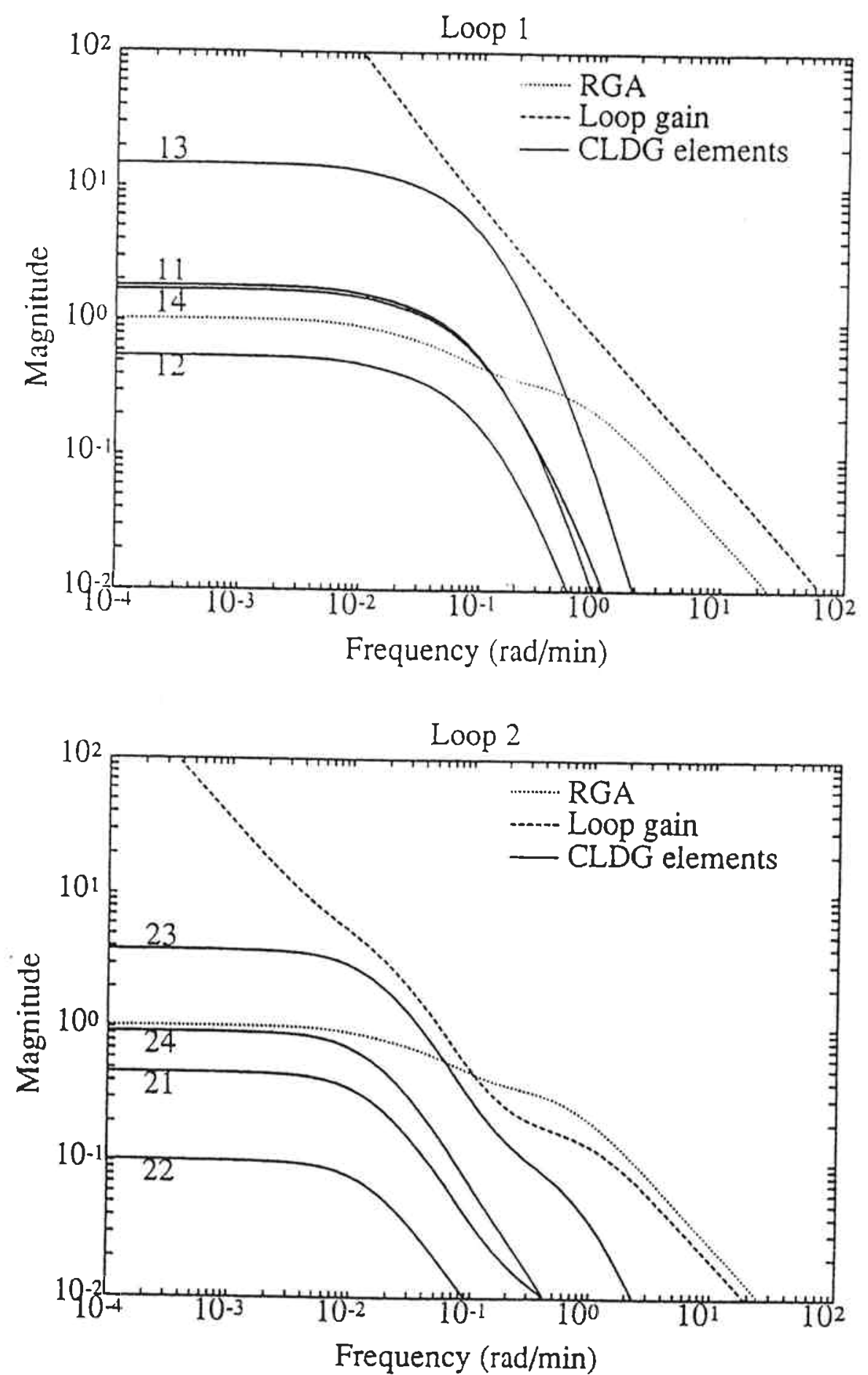

Figure 9. Closed loop disturbance gains, $\delta_{i k}$, relative gain $\lambda_{i i}$ and loop gain $g_{i i} c_{i}$ for FCC example. 


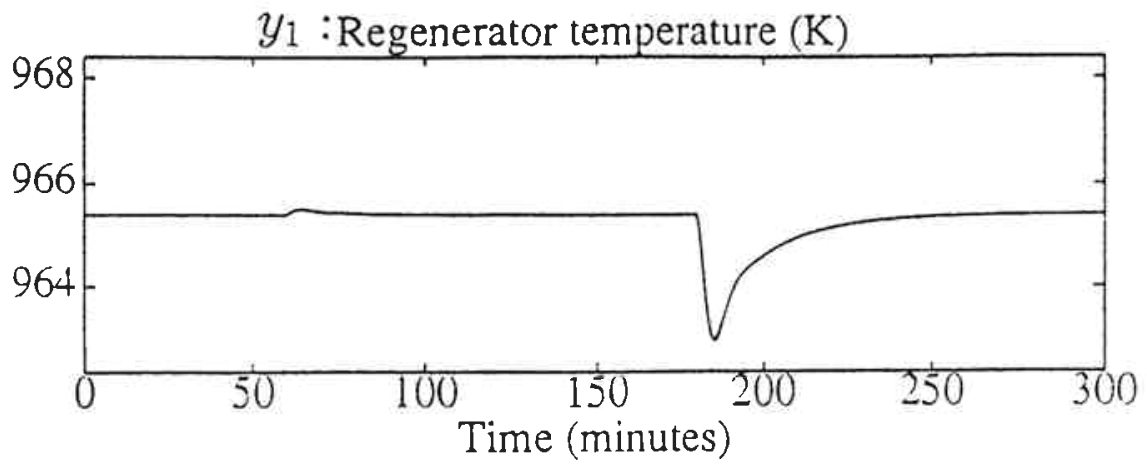

$y_{2}:$ Temperature rise dense bed-cyclones $(K)$
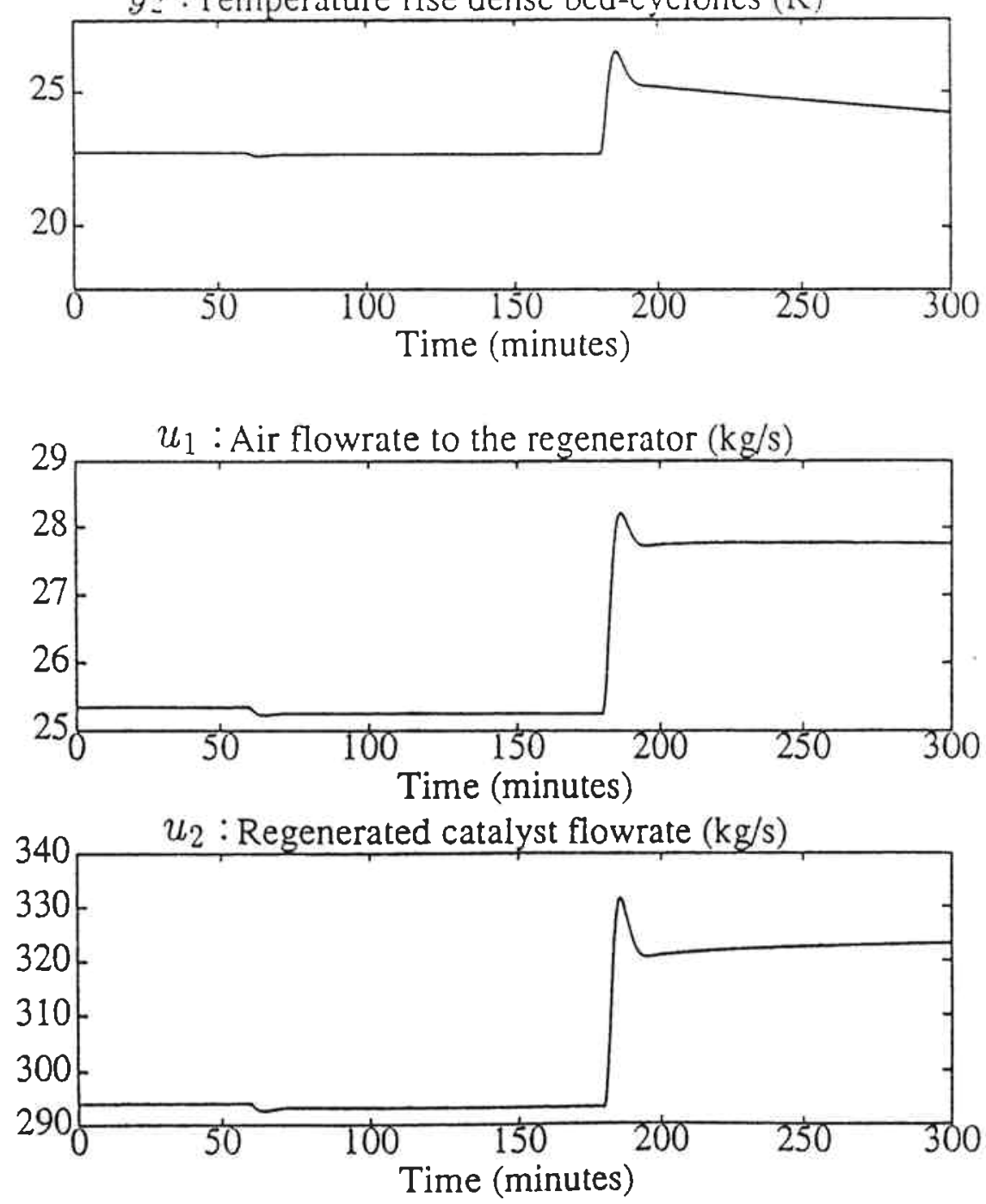

Figure 10. Disturbance rejection for FCC example using Kurihara control structure. Responses to a unit step in $z_{2}$ at $t=60 \mathrm{~min}$ and a unit step in $z_{3}$ at $t=180 \mathrm{~min}$. 
Generalizations are also possible. Consider controllers on the form $C(s)=H(s) K(s)$ where $K(s)$ is a diagonal matrix (for example, consisting of PID controllers), and $H(s)$ is a multivariable precompensator, for example, a constant matrix (steady-state or highfrequency decoupler), a simple dynamic decoupler or a one-way decoupler. The performance results on decentralized control may be generalized to include decouplers, $H(s)$, by replacing $G$ by $G H$ when evaluating PRGA and CLDG.

\section{ACKNOWLEDGMENT}

Support from NTNF is gratefully acknowledged.

\section{Nomenclature}

$\boldsymbol{e}=y-r \quad$ vector of control errors

$g_{i j}=[G]_{i j}$ ijth element of $\mathrm{G}$

$G(s)$ plant model for effect of $u$ on $y$

$G_{\text {diag }}(s)$ matrix consisting of diagonal elements of $G$

$g_{d i k}=\left[G_{d}\right]_{i k}$ ikth element of $G_{d}$

$G_{d}(s)$ disturbance model for effect of $z$ and $y$

$r$ vectors of reference outputs (setpoints)

$S=(I+G C)^{-1}$ sensitivity function

$u$ vector of manipulated inputs

$y$ vector of outputs

$z \quad$ vector of disturbances

$\beta_{i k} \quad i k$ th elements of RDG matrix (Stanley et al., 1985)

$\delta_{i k}(s)=g_{i i}(s)\left[G^{-1}(s) G_{d}(s)\right]_{i k} \quad$ Closed Loop Disturbance Gain (CLDG)

$\Delta(s)=G_{\text {diag }}(s) G^{-1} G_{d}(s) \quad$ CDLG-matrix

$\gamma_{i j}(s)=g_{i i}(s)\left[G^{-1}(s)\right]_{i j} \quad i j$ th element in PRGA matrix

$\Gamma(s)=G_{\text {diag }}(s) G^{-1}(s) \quad$ PRGA matrix

$\lambda_{i j}(s)=g_{i j}(s)\left[G^{-1}(s)\right]_{j i} \quad$ ijth element in RGA matrix

$\Lambda(s) \quad$ RGA matrix

$\omega$ frequency [ $\mathrm{rad} / \mathrm{min}]$

$\omega_{B}$ closed loop bandwidth, frequency at which asymptote of $|S(j \omega)|$ first reaches 1

\section{Subscripts}

$i$ index for output or loops

$j$ index for manipulated inputs or setpoints

$k$ index for disturbances.

\section{REFERENCES}

BalCHEN, J. G. (1958). Lecture Notes in Control (Norwegian Institute of Technology (NTH), Norway (in Norwegian).

BRISTOL, E. H. (1966). On a new measure of interactions for multivariable process control, IEEE Trans. Automat. Control, AC-11, 133-134. 
BRistol, E. H. (1978). Recent results on interactions in multivariable process control, AIChE Annual Meeting, Chicago.

FrIEDLY, E. H. (1984). Use of the Bristol array in designing noninteracting control loops. A limitation and extension, Ind. Eng. Chem. Process Des. Dev., 23, 469-472.

GrosDidiER, P. (1990), Analysis of interaction direction with the singular value decomposition, Computers \& Chem. Engng., 14, 687-689.

Hovd, M., LUNDSTRÖM, P. and SKogesTAD, S. (1990). Controllability analysis using frequencydependent tools for interactions and disturbances, AIChE Annual Meeting, Chicago, Paper 312j.

Hovd, M. and SkOGESTAD, S. (1992). Use of simple frequency-dependent tools for control system analysis, structure selection and design. Automatica (in press).

Kurihara, H. (1967). Optimal Control of Fluid Catalytic Cracking Processes, MIT Electr. Sys. Lab., report ESL-R-309.

LeE, E. and GroveS, F. R., JR. (1985). Mathematical model of the fluidized bed catalytic cracking plant, Trans. Soc. Comp. Sim., 2, 219-236.

McAvoy, T. J. (1983). Interaction Analysis (Instrument Society of America, Research Triangle Park, USA).

Morari, M. (1983). Design of resilient processing plants-III, Chem. Eng. Sci., 38, 1881-1891.

PERKINS, J. D. (1989). Interactions between process design and process control, Preprints IF AC Symposium DYCORD +89 (Maastricht, NL).

RosenBrock, H. H. (1970). State-Space and Multivariable Theory (Nelson, London).

Seborg, D. E., Edgar, T. F. and Mellichamp, D. A. (1989). Process Dynamics and Control, (Wiley, New York).

Shinskey, F. G. (1967), Process Control Systems, (McGraw-Hill, New York).

ShINSKEY, F. G. (1984). Distillation Control, 2nd Edition (McGraw Hill, New York).

Skogestad, S. and Hovd, M. (1990). Use of frequency-dependent RGA for control structure selection, Proc. American Control Conference, San Diego, 2133-2139.

Skogestad, S. and LundSTRÖM, P. (1990). Mu-optimal LV-control of distillation columns, Computers \& Chem. Engng., 14, 401-413.

Skogestad, S. and Morari, M. (1987 a). Effect of disturbance directions on closed loop performance, Ind. Eng. Chem. Res., 26, 2029-2035.

Skogestad, S. and Morari, M. (1987 b). Implications of large RGA elements on control performance, Ind. Eng. Chem. Res., 26, 2323-2330.

Skogestad, S., Lundström, P. and JACOBSEN, E. W. (1990). Selecting the best distillation control configuration, AIChE Journal, 36, 753-764.

Stanley, G., Marino-Gallarraga, M., McAvoy, T. J. (1985). Shortcut operability analysis. 1. The relative disturbance gain. Ind. Eng. Chem. Process Des. Dev., 24, 1181-1188.

Stephanopoulos, G. (1984). Chemical Process Control (Prentice-Hall, New York). 